\title{
RELAÇÃO HIPSOMÉTRICA PARA Araucaria angustifolia (Bert.) O. Ktze NA REGIÃO OESTE DO ESTADO DO PARANÁ
}

\author{
Hypsometric relation for Araucaria angustifolia (Bert.) \\ O. Ktze In West of Parana
}

\author{
Marcos Vinicius Winckler Caldeira* \\ Mauro Valdir Schumacher" \\ Luciano Weber Scheeren"** \\ Luciano Farinha Watzlawick
}

\section{Resumo}

Este trabalho teve como objetivos selecionar modelos matemáticos para estimar a altura das árvores em função do diâmetro à altura do peito (DAP), em povoamentos de Araucária, Araucaria angustifolia (Bert.) O. Ktze, na região Oeste do Estado do Paraná. Para o ajuste dos dados de altura foram testadas 17 equações matemáticas, sendo utilizadas equações lineares aritméticas e logarítmicas. Como critérios estatísticos de seleção das melhores equações foi utilizado o coeficiente de determinação ajustado $\left(\mathrm{R}_{\mathrm{aj}}\right)$, o erro padrão de estimativa $\left(\mathrm{S}_{\mathrm{yx}}\right)$, o coeficiente de variação em percentagem (CV\%), o índice de Furnival em percentagem (IF\%) e o valor da estatística F. Em seguida foi realizada a análise gráfica dos resíduos para os cinco melhores modelos matemáticos. A equação 17 , representada $\mathrm{h}=\left(\mathrm{d} / \mathrm{b}_{0}+\mathrm{b}_{1}{ }^{*} \mathrm{~d}\right)^{2}$ resultou como modelo mais eficiente, ajustado em função do DAP das árvores, para estimar as alturas em povoamentos com idade de 14 anos de idade.

Palavras-chave: Relação hipsométrica, Altura, Diâmetro, Araucaria.

* Engenheiro Florestal, Doutorando em Ciências Florestais, Curso de Pós-Graduação em Engenharia Florestal Centro de Ciências Florestais e da Madeira, UFPR. Bolsista do CNPq.

Rua Pref. Lothário Meissner, n. ${ }^{\circ}$ 3400, Jardim Botânico, Curitiba - PR, CEP 80210-170.

E-mail caldeira@floresta.ufpr.br.

** Engenheiro Florestal, Dr. Prof. Adjunto do Departamento de Ciências Florestais, Centro de Ciências Rurais, UFSM. schuma@ccr.ufsm.br 3.Eng ${ }^{\circ}$ Florestal, Doutorando em Ciências Florestais, Curso de Pós-Graduação em Engenharia Florestal, UFSM. Santa Maria, RS.

**** Engenheiro Florestal, Doutorando em Ciências Florestais, Curso de Pós-Graduação em Engenharia Florestal, UFSM. Santa Maria, RS.

**** Engenheiro Florestal, Doutorando em Ciências Florestais, Curso de Pós-Graduação em Engenharia Florestal Centro de Ciências Florestais e da Madeira, UFPR. 


\section{Abstract}

This work had as objectives to select mathematical models to predict the height of trees in function of diameter at the brust height (DBH), in Araucaria stands, Araucaria angustifolia (Bert.) O. Ktze, in West region of Paraná State. For the adjustment of the height data 17 mathematical equations were tested, being used arithmetic and logarithmic linear equations. Were used 5 statistical criterion for the best equations selection: a) adjusted determination coefficient (Raj), b) standard error of estimate (Syx), c) variation coefficient in percentage (CV\%), d) Furnival index in percentage (IF\%) and e) F statistic. The residues graphic analysis was accomplished for the five better mathematical models. The equation 17 , represented by $\mathrm{h}=(\mathrm{d} / \mathrm{b} 0+\mathrm{b} 1 * \mathrm{~d})^{2}$ resulted as more efficient model, adjusted in function of the trees DAP, to adjust the heights in stands with 14 year-old age.

Keywords: Hipsometric relation; Height; Diameter; Araucaria.

\section{Introdução}

A Araucaria angustifolia é uma espécie nativa, conhecida como pinheiro-do-paraná e/ou pinho, pinheiro-brasileiro. É uma árvore alta, de aspecto original e contrastante com as demais árvores do sul do Brasil, de 20-50 metros de altura, 1-2 metros de diâmetro na altura do peito. O tronco é perfeitamente cilíndrico, reto e raras vezes ramificado em dois ou mais, casca grossa e resinosa, cuja superfície externa se desprende em placas cinzento-escuras (REITZ et al., 1983). Apresenta madeira de inúmeras utilizações e crescimento médio, quando comparada a espécies exóticas, sendo considerada a conífera de maior expressão econômica no Brasil.

Dentre as árvores do sul do Brasil, a Araucaria angustifolia foi a que mais beneficiou o homem. Foi esta espécie, no Rio Grande do Sul, a primeira matéria-prima que proporcionou grandes acúmulos de capital àqueles que se aventuraram a sua exploração. Estima-se, segundo Gerhardt (1999), que na época apenas 23\% das árvores abatidas eram aproveitadas, ficando o restante na floresta. É possível, ainda hoje, encontrar restos de árvores gigantescas, na forma de nós nos campos que testemunharam uma época de fartura, não muito distante.
Durante décadas, a Araucaria angustifolia foi a espécie economicamente mais importante do Brasil, além disso, ocupou, até o início dos anos 70, lugar importante na economia exportadora do Brasil. Em função do abuso no consumo desse recurso nacional, bem como a expansão de áreas de uso agrícola, foram rapidamente reduzidas as áreas com floresta de Araucaria, que originalmente cobriam cerca de $200.000 \mathrm{~km}^{2}$ (CARVALHO, 1994). No entanto, em 1963, essa área alcançava somente cerca de $30.000 \mathrm{~km}^{2}$ e desde então vem diminuindo mais ainda (HUECK, 1972).

A ocorrência da espécie se dá principalmente nos estados do Paraná, Santa Catarina e Rio Grande do Sul, com 40, 31, e 25\% de sua superfície total, respectivamente. Os demais $4 \%$ da cobertura original de Araucária encontravam-se nos estados de São Paulo, Minas Gerais e Rio de Janeiro (CARVALHO, 1994). No Brasil, a sua ocorrência limita-se entres as latitudes de $15^{\circ}$ e $30^{\circ}$ Sul, e longitude $43^{\circ} 30^{\prime}$ e $57^{\circ} 30^{\prime}$ Oeste, apesar de existir uma distribuição irregular (HUECK, 1972).

Em função da importância da Araucaria angustifolia e a necessidade de implantar o planejamento florestal, torna-se necessário estudar as relações biométricas, dentre as quais a relação hipsométrica, pois isso possibilita conhecer com precisão a altura das árvores indiretamente, reduzindo o tempo e o custo do levantamento de dados no inventário florestal. Neste sentido, foram testados vários modelos matemáticos para o ajuste de alturas sobre diâmetros em povoamentos de Araucaria angustifolia, aos 14 anos de idade, plantadas na região de Quedas do Iguaçu, PR. O objetivo foi selecionar o melhor modelo de relação hipsométrica no primeiro desbaste da espécie, em função dos diâmetros medidos a altura do peito.

\section{Revisão bibliográfica}

A relação hipsométrica, conforme Scolforo (1997), é uma opção de trabalho controvertida, mas de grande utilização. É um aspecto de suma importância a ser considerado na coleta das informações, sendo de grande utilidade prática principalmente em populações com árvores de grande porte.

Concernente, especificamente, à relação altura e diâmetro, pode-se considerar, de acordo com Scolforo (1997), duas situações: a primeira se 
refere a povoamentos em sítios bem definidos, bem formados e conduzidos; nesta situação espera-se uma correlação alta entre as variáveis diâmetro e altura, pois haverá maior homogeneidade na população considerada. Em uma segunda situação, em povoamentos mais antigos, malformados ou malconduzidos, ou em sítios não muito bem definidos, neste caso, espera-se uma correlação baixa entre as variáveis diâmetro e altura. Haverá, neste caso, uma maior heterogeneidade na população considerada.

A relação hipsométrica é influenciada pela idade, sítio, densidade, posição sociológica, tamanho da copa e espécie (LOETSCH et al., 1973; HUSCH et al., 1982; FINGER, 1992; SCOLFORO, 1999). Em sítios bons, as melhores condições locais aceleram o crescimento, favorecendo a formação de classes sociológicas. Em conseqüência, povoamentos jovens, em sítios bons, mostram uma curva de altura íngreme, enquanto em sítios pobres as curvas para uma mesma espécie são mais achatadas (HUSCH et al., 1982).

A idade do povoamento afeta a relação entre diâmetro e altura, portanto, em inventários sucessivos não se deve utilizar a mesma relação e sim refazê-la a partir de novos dados (SCOLFORO, 1999). À medida que aumenta a idade do povoamento, as diferenças entre as curvas tendem a diminuir gradativamente, pois quando a árvore atinge seu clímax de crescimento, sendo isso uma característica da espécie e sítio, o seu crescimento em diâmetro e altura será reduzido e, como conseqüência, as mudanças na relação entre H e D serão muito pequenas (FINGER, 1992).

No que se refere à densidade, segundo Finger (1992), essa influência será maior ou menor, dependendo de qual estrato da floresta a árvore pertence. Nas árvores dominantes, a altura é pouco afetada pelo espaçamento, enquanto que nas dominadas a influência do crescimento em altura é bastante acentuada. Concernente à variável diâmetro, esta é bastante afetada em qualquer estrato, em função da concorrência. Neste sentido, quando a concorrência for alta, a razão H/D será maior que a concorrência mais moderada.

O comprimento da copa também influencia a relação hipsométrica, pois quanto maior a copa menor será a razão H/D (SCOLFORO, 1999).

Segundo Sterba (1986), citado por Zanon et al. (1996), a análise dos coeficientes da função de regressão, utilizada para descrever a relação entre a altura e o diâmetro, tem evidenciado que, com o aumento da idade, os coeficientes angulares apresentam somente uma variação ao acaso, enquanto o intercepto da função tende a diminuir. Assim, torna-se possível estimar o valor do intercepto para diferentes sítios naturais, mantendo o valor do ângulo de inclinação constante.

Muitas vezes, um modelo é ajustado com dados de diferentes estratos, podendo ser utilizada, por exemplo, a estratificação por idade, espaçamento e/ou sítios. Uma pergunta que sempre surge, segundo Azevedo et al. (1999), é se realmente seria necessária uma equação independente para cada um desses estratos. O fato é que, em muitos casos, uma única equação obtida, por meio de dados agrupados, poderá ser utilizada como estimativa comum para todos os estratos. No entanto, uma vez escolhido o melhor modelo para representar os dados é apropriado averiguar, ainda, se uma determinada equação, gerada por um conjunto de dados "a", poderá ser usada em detrimento de uma outra, obtida em um conjunto de dados "b", ou ainda, se será melhor usar uma terceira equação, obtida com os dados "a" e "b".

\section{Material e métodos}

O presente trabalho foi realizado em um povoamento de Araucaria angustifolia, aos 14 anos de idade, pertencente à empresa Araupel S.A., localizada na cidade de Quedas do Iguaçu/PR. A área encontra-se entre as coordenadas geográficas 25 e $26^{\circ}$ latitude Sul e $52^{\circ}$ e $53^{\circ}$ de longitude Oeste de Greenwich.

Pelo sistema de Köppen, o clima é subtropical úmido mesotérmico. A temperatura média do trimestre mais quente é de $23,0^{\circ} \mathrm{C}$, temperatura média do trimestre mais frio é de $15,5^{\circ} \mathrm{C}$ e a temperatura média anual é de $19,5^{\circ} \mathrm{C}$. A temperatura mínima absoluta é de $-5,5^{\circ} \mathrm{C}$, já a temperatura máxima absoluta chega a $38,1^{\circ} \mathrm{C}$. A precipitação pluvial média do ano é de $1900 \mathrm{~mm}$. Os meses mais chuvosos vão de outubro a dezembro, enquanto que os meses de menor precipitação são março, julho e agosto (MORENO, 1961).

O povoamento encontra-se em um Latossolo Roxo, com um horizonte A bem desenvolvido, com teores médios de cálcio e magnésio, profundo, friável, poroso, bem drenado e com boa capacidade de retenção de água, textura franca a 
argilosa. Na Tabela 1, são apresentadas as características químicas do solo.

O povoamento de Araucária foi implantado entre julho a agosto de 1986. No momento da coleta dos dados, apresentava-se com 14 anos de idade, sendo o espaçamento inicial de 3,0 $\mathrm{m} \mathrm{x}$ $0,5 \mathrm{~m}$, totalizando 6.666 plantas por hectare. As sementes, utilizadas para o plantio, foram coletadas nas florestas nativas, localizadas no Município de Irineópolis, SC. O sistema de plantio utilizado foi o mecanizado, com plantadoras especiais, desenvolvidas pela própria empresa. Com estas, foi possível plantar o pinhão diretamente no solo e no local definitivo.

TABELA 1 - Análise química do solo da área com Araucaria angustifolia onde foi realizada a amostragem de serapilheira e biomassa acima do solo

Table 1 - Soil chemical analysis of an area with Araucaria angustifolia where it was carried out a litter and biomass sampling above of the ground

\begin{tabular}{cccccc}
\hline $\begin{array}{c}\text { Prof. } \\
(\mathrm{cm})\end{array}$ & $\begin{array}{c}\text { Argila } \\
(\%)\end{array}$ & $\begin{array}{c}\mathrm{pH} \\
\mathrm{H}_{2} \mathrm{O}\end{array}$ & $\begin{array}{c}\mathrm{M} . \mathrm{O} \\
\left(\mathrm{g} \mathrm{kg}^{-1}\right)\end{array}$ & $\mathrm{P}$ & $\mathrm{K}$ \\
\hline $0-20$ & 81 & 4,2 & 37 & 1,1 & 80,0 \\
\hline $\mathrm{Ca}$ & $\mathrm{Mg}$ & $\mathrm{Al}$ & $\mathrm{H}+\mathrm{Al}$ & $\mathrm{CTC}$ & $\left.\mathrm{V} \mathrm{mg}^{-1}\right)$ \\
& \multicolumn{2}{c}{$\left(\mathrm{cmol}_{\mathrm{c}} \mathrm{L}^{-1}\right)$} & & $\%$ \\
2,2 & 0,7 & 2,9 & 12,2 & 5,9 & 20,6 \\
\hline
\end{tabular}

No primeiro ano, nas entrelinhas da Araucária foi plantado arroz de sequeiro. O arroz foi adubado com $250 \mathrm{~kg}$ plantas por hectare de $\mathrm{N}_{2} \mathrm{P}_{2} \mathrm{O}_{5}-$ $\mathrm{K}_{2} \mathrm{O}$ na fórmula 4-20-20, o que equivale a uma adubação com $10 \mathrm{~kg} \mathrm{ha}^{-1}$ de N, $50 \mathrm{~kg} \mathrm{ha}^{-1}$ de $\mathrm{P}_{2} \mathrm{O}_{5}$ e 50 $\mathrm{kg} \mathrm{ha}^{-1}$ de $\mathrm{K}_{2} \mathrm{O}$. No segundo e terceiro ano foi plantado milho entre as fileiras de araucária, porém, sem adubação. Não foi realizado nenhum replantio, uma vez que havia um elevado número de plantas na fase inicial.

Nos primeiros 3 anos, após o plantio, foram necessários algumas práticas de manutenção do povoamento, com o objetivo de garantir o estabelecimento, bom crescimento e desenvolvimento das plantas. Foram realizadas as práticas de combate a formigas, coroamento, capina manual, roçada manual, aplicação manual e mecanizada de herbicida e roçada de arbustos.

Em relação aos tratos culturais, o raleio teve por objetivo estabelecer a população ideal para o povoamento de Araucária, por meio de eliminação de indivíduos de menor potencial, buscando-se a formação de espaços homogêneos entre as plantas, próximos a $6 \mathrm{~m}$ " (espaçamento $3 \mathrm{~m} \times 2 \mathrm{~m}$ ).
A densidade inicial (6.666 plantas/ha) exigiu, a partir do segundo para o terceiro ano, uma operação de redução do número de indivíduos por hectare a fim de possibilitar o crescimento ideal e contínuo das plantas remanescentes. Tendo como base o espaçamento $3 \mathrm{~m} \times 2 \mathrm{~m}$, reduziu-se o número de plantas para 1.666 plantas por hectare.

O povoamento sofreu a intervenção de 3 podas: a) poda baixa: realizada no ano de 1989, após o raleio, em todas as árvores do povoamento. Esta poda atingiu uma altura média de 2,0 a 2,5 m; b) poda média: realizada no ano de 1991, também em todas as árvores do povoamento. Esta poda atingiu uma altura média em torno de 4,0 a 4,50 m; e c) poda alta: realizada no ano de 1993, de forma seletiva, ou seja, em mais ou menos 400 a 500 árvores por hectare, as quais deveriam permanecer até o corte final, por volta de 30 a 35 anos. Esta poda atingiu uma altura média de $6,50 \mathrm{~m}$.

Com base nas medições do DAP e altura, foram ajustados modelos de relação hipsométrica, apresentados na Tabela 2, visando a selecionar a melhor equação para o ajuste dos dados. 
TABELA 2 - Equações de regressão testadas para estimar as alturas de Araucaria angustifolia

Table 2 - Equations used for regression analysis to estimate heights of Araucaria angustifolia

\begin{tabular}{|c|c|}
\hline Equação & Modelo matemático \\
\hline $1 \ln \mathrm{h}=\mathrm{b}_{\mathrm{o}}+\mathrm{b}_{1} * 1 / \mathrm{d}$ & $9 \mathrm{~h}=\mathrm{b}_{\mathrm{o}}+\mathrm{b}_{1} * \mathrm{~d}+\mathrm{b}_{2} * \mathrm{~d}^{2}$ \\
\hline $2 \ln \mathrm{h}=\mathrm{b}_{\mathrm{o}}+\mathrm{b}_{1} * \mathrm{~d}^{2}$ & $10 \mathrm{~h}=1 /\left(\mathrm{b}_{0}+\mathrm{b}_{1} * 1 / \mathrm{d}^{2}\right)+1,30$ \\
\hline $3 \ln \mathrm{h}=\mathrm{b}_{\mathrm{o}}+\mathrm{b}_{1} * 1 / \mathrm{d}+\mathrm{b}_{2} * 1 / \mathrm{d}^{2}$ & $11 \log h=b_{0}+b_{1} * 1 / d$ \\
\hline $4 \ln \mathrm{h}=\mathrm{b}_{\mathrm{o}}+\mathrm{b}_{1} * \ln \mathrm{d}$ & $12 \mathrm{~h}=\mathrm{b}_{\mathrm{o}}+\mathrm{b}_{1} * 1 / \mathrm{d}^{2}$ \\
\hline $5 \mathrm{~h}=\mathrm{b}_{\mathrm{o}}+\mathrm{b}_{1} * \ln \mathrm{d}$ & $13 \ln (h-1,30)=b_{0}+b_{1} * \ln d^{2}$ \\
\hline $6 \mathrm{~h}=\mathrm{b}_{\mathrm{o}}+\mathrm{b}_{1} * 1 / \mathrm{d}$ & $14 \log h=b_{o}+b_{1} * \log d$ \\
\hline $7 \mathrm{~h}=\mathrm{b}_{\mathrm{o}}+\mathrm{b}_{1} * \mathrm{~d}^{2}$ & $15 \mathrm{~h}-1,30=\mathrm{d}^{2} /\left(\mathrm{b}_{0}+\mathrm{b}_{1} * \mathrm{~d}+\mathrm{b}_{2} * \mathrm{~d}^{2}\right)$ \\
\hline $8 \mathrm{~h}=\mathrm{b}_{\mathrm{o}}+\mathrm{b}_{1} * 1 / \mathrm{d}+\mathrm{b}_{2} * \mathrm{~d}^{2}$ & $16 \mathrm{~h}=\mathrm{d}^{2} /\left(\mathrm{b}_{0}+\mathrm{b}_{1} * \mathrm{~d}+\mathrm{b}_{2} * \mathrm{~d}^{2}\right)$ \\
\hline & $17 \mathrm{~h}=\left(\mathrm{d} / \mathrm{b}_{0}+\mathrm{b}_{1} * \mathrm{~d}\right)^{2}$ \\
\hline
\end{tabular}

Onde: $\mathrm{h}=$ altura total em metros; $\mathrm{d}=$ diâmetro à a ltura do peito (DAP) em centímetros; $\mathrm{ln}=1$ logaritmo neperiano; $\log =\operatorname{logaritmo}$ na base $10 ; b_{o}, b_{1}, b_{2}, b_{3}$, = coeficientes de regressão

Numa área de condições de sítio semelhantes, foram demarcadas 7 parcelas com as dimensões de $20 \mathrm{~m}$ x $25 \mathrm{~m}$. Em cada uma destas, foram medidos todos os diâmetros das árvores (DAP) e 10\% de suas alturas.

A partir do ajuste dos dados, foi realizada a seleção dos cinco melhores modelos matemáticos, com base nos seguintes critérios estatísticos: a) coeficiente de determinação ajustado $\left(\mathrm{R}_{\mathrm{aj}}\right)$; b) erro padrão da estimativa $\left(\mathrm{S}_{\mathrm{yx}}\right)$; c) coeficiente de variação em percentagem (Č $\%)$; d) Índice de Furnival em percentagem (IF\%); e) valor da estatística F. Em seguida, foi realizada a análise gráfica dos resíduos para os cinco melhores modelos matemáticos.

A utilização das estatísticas CV\% e IF\% é necessária devido a estarem sendo comparados modelos matemáticos aritméticos, que sofreram transformação logarítmica da variável dependente (altura). Dessa forma, é necessário a aplicação do Índice de Furnival em percentagem (IF\%), calculado para os modelos logarítmicos, que permitirá a comparação com o valor do coeficiente de variação em percentagem (CV\%), calculado para modelos aritméticos (SCHNEIDER, 1993).

Conforme os critérios apresentados acima, as melhores equações deverão apresentar, simultaneamente, o maior coeficiente de determinação ajustado, o menor erro padrão da estimativa e o menor coeficiente de variação em percentagem, ou o melhor Índice de Furnival em percentagem e maior valor da estatística $F$.

\section{Resultados e discussão}

Com o processamento das equações matemáticas, foram obtidos os parâmetros estatísticos que permitiram selecionar os três modelos matemáticos com melhor ajuste aos dados. A Tabela 3 inclui os coeficientes $\left(b_{0}, b_{1}\right.$ e $\left.b_{2}\right)$, o Coeficiente de Determinação ajustado $\left(R_{a j}^{2}\right)$, o erro padrão de estimativa $\left(\mathrm{S}_{\mathrm{vx}}\right)$, o Coeficiente de Variação em percentagem (CV\%), o Índice de Furnival em percentagem $\left(\mathrm{R}_{\text {aj }}^{2}\right)$ e a estatística $(\mathrm{F})$. 
TABELA 3 - Parâmetros estatísticos para as equações testadas

Table 3 - Statistical parameters of the tested equations

\begin{tabular}{|c|c|c|c|c|c|c|c|c|}
\hline \multirow[t]{2}{*}{ Modelo } & \multicolumn{3}{|c|}{ Coeficientes } & \multirow[t]{2}{*}{$\mathrm{R}^{2}$} & \multirow{2}{*}{$S_{y x}$} & \multirow{2}{*}{$\mathrm{CV} \%$} & \multirow{2}{*}{$\mathrm{IF} \%$} & \multirow{2}{*}{$\mathrm{F}$} \\
\hline & $\mathrm{b}_{0}$ & $\mathrm{~b}_{1}$ & $\mathrm{~b}_{2}$ & & & & & \\
\hline 1 & 3,135 & $-8,029$ & - & 0,40 & 0,10 & - & 52,30 & 48,3 \\
\hline 2 & 2,330 & 0,001 & - & 0,40 & 0,10 & - & 52,30 & 48,5 \\
\hline 3 & 3,372 & $-15,060$ & 50,817 & 0,40 & 0,10 & - & 52,30 & 24,1 \\
\hline 4 & 1,120 & 0,544 & - & 0,41 & 0,10 & - & 52,30 & 49,6 \\
\hline 5 & $-6,032$ & 7,207 & - & 0,42 & 1,26 & 9,14 & - & 51,7 \\
\hline 6 & 20,628 & $-105,796$ & - & 0,41 & 1,27 & 9,21 & - & 49,5 \\
\hline 7 & 9,958 & 0,015 & - & 0,42 & 1,26 & 9,14 & - & 52,4 \\
\hline 8 & 13,123 & $-32,019$ & 0,011 & 0,42 & 1,27 & 9,21 & - & 26,1 \\
\hline 9 & 7,420 & 0,328 & 0,005 & 0,42 & 1,27 & 9,21 & - & 26,0 \\
\hline 10 & 0,058 & 5,471 & - & 0,39 & 0,01 & 0,07 & - & 45,4 \\
\hline 11 & 1,362 & $-3,486$ & - & 0,40 & 0,04 & - & 20,92 & 48,3 \\
\hline 12 & 16,985 & $-746,403$ & - & 0,39 & 1,29 & 9,36 & - & 46,3 \\
\hline 13 & 0,854 & 0,302 & - & 0,41 & 0,11 & - & 57,53 & 49,3 \\
\hline 14 & 0,486 & 0,544 & - & 0,41 & 0,04 & - & 20,92 & 49,6 \\
\hline 15 & $-8,528$ & 1,921 & $-0,006$ & 0,78 & 2,12 & 15,37 & - & 123,9 \\
\hline 16 & $-7,534$ & 1,640 & $-0,0001$ & 0,83 & 1,72 & 12,47 & - & 169,8 \\
\hline 17 & 1,166 & 0,196 & - & 0,83 & 0,20 & 1,45 & - & 345,9 \\
\hline
\end{tabular}

Na Tabela 4, pode-se observar as 5 melhores equações matemáticas, selecionadas para a análise gráfica dos resíduos em percentagem.

Devido as equações apresentarem diferente número de coeficientes, a análise da variação total dos dados deve ser realizada com base no coeficiente de determinação, ajustado para o número de coeficientes de cada modelo matemático. Observa-se que duas das equações selecionadas apresentaram um coeficiente de determinação ajustado em torno de 0,40 e três delas um valor em torno de 0,80 , o que demonstra que a variação total dos dados foi bem explicada apenas por três equações (Tabela 4). 
TABELA 4 - Equações de regressão selecionadas para análise gráfica dos resíduos em percentagem Table 4 - Selected regression equations for residues graphical analysis in percentage

\begin{tabular}{clccccc}
\hline Equação & \multicolumn{1}{c}{ Modelo matemático } & $\mathrm{R}_{\mathrm{aj}}^{2}$ & $\mathrm{~S}_{\mathrm{yx}}$ & $\mathrm{CV} \%$ & $\mathrm{IF} \%$ & $\mathrm{~F}$ \\
\hline 5 & $\mathrm{~h}=\mathrm{b}_{\mathrm{o}}+\mathrm{b}_{1} * \ln \mathrm{d}$ & 0,42 & 1,26 & 9,14 & - & 51,7 \\
7 & $\mathrm{~h}=\mathrm{b}_{\mathrm{o}}+\mathrm{b}_{1} * \mathrm{~d}^{2}$ & 0,42 & 1,26 & 9,14 & - & 52,4 \\
15 & $\mathrm{~h}-1,30=\mathrm{d}^{2} /\left(\mathrm{b}_{0}+\mathrm{b}_{1} * \mathrm{~d}+\mathrm{b}_{2} * \mathrm{~d}^{2}\right)$ & 0,78 & 2,12 & 15,37 & - & 123,9 \\
16 & $\mathrm{~h}=\mathrm{d}^{2} /\left(\mathrm{b}_{0}+\mathrm{b}_{1} * \mathrm{~d}+\mathrm{b}_{2} * \mathrm{~d}^{2}\right)$ & 0,83 & 1,72 & 12,47 & - & 169,8 \\
17 & $\mathrm{~h}=\left(\mathrm{d} / \mathrm{b}_{0}+\mathrm{b}_{1} * \mathrm{~d}\right)^{2}$ & 0,83 & 0,20 & 1,45 & - & 345,9 \\
\hline
\end{tabular}

A análise da dispersão, entre os valores observados e estimados pela análise de regressão, para os modelos aritméticos, é feita com base no erro padrão de estimativa $\left(\mathrm{S}_{\mathrm{yx}}\right)$, quando os modelos matemáticos apresentam variáveis dependentes de mesma unidade, ou no coeficiente de variação em percentagem (CV\%), quando os modelos matemáticos apresentam variáveis dependentes de diferentes unidades. Para comparar a eficiência dos modelos matemáticos, que apresentam a variável dependente transformada para logaritmo, utiliza-se a estatística Índice de Furnival em percentagem (IF\%). Dessa forma, a comparação e seleção de modelos matemáticos, aritméticos e logarítmicos, deve ser realizada utilizando-se CV\% e no IF\%, pois o erro padrão de estimativa $\left(S_{y x}\right)$ não constitui uma estatística eficiente nesses casos. Nestas circunstâncias, todos os modelos selecionados eram aritméticos.

Apesar da equação 14 apresentar um $\mathrm{S}_{\mathrm{yx}}$ bastante inferior às demais, não se pode afirmar que esta apresenta uma menor dispersão entre os valores observados e estimados, sendo necessário comparar o valor do IF\% com o CV\% das demais equações (Tabela 3).

Com respeito à estatística F, esta se mostrou bastante elevada apenas na equação 17 (Tabela 4). Considerando a utilização de 70 conjuntos de dados de altura e idade e que a significância de cada modelo matemático é dada pela divisão de 1 grau de liberdade do modelo matemático pelo valor da respectiva estatística F, todos os modelos resultaram altamente significativos, sendo que o modelo 5 apresenta o nível de significância mais baixo, com $0,02(1 / 51,7)$ e o modelo 17 mostra-se mais significativo, com valor de $0,003(1 / 345,9)$.

Nas Figuras 1 a 5 podem ser observados os resíduos entre a altura observada e a altura estimada pelas respectivas equações, em função do diâmetro à altura do peito (DAP). Observa-se, pela análise dos gráficos, que a equação 15 apresenta uma tendência de subestimar a altura nas árvores em toda a amplitude de diâmetros, observados (DAP). As demais equações não apresentaram qualquer tipo de tendências.

FIGURA 1 - Resíduos para altura observada e estimada com a equação 5

Figure 1 - Residues for the observed and estimated height using equation 5

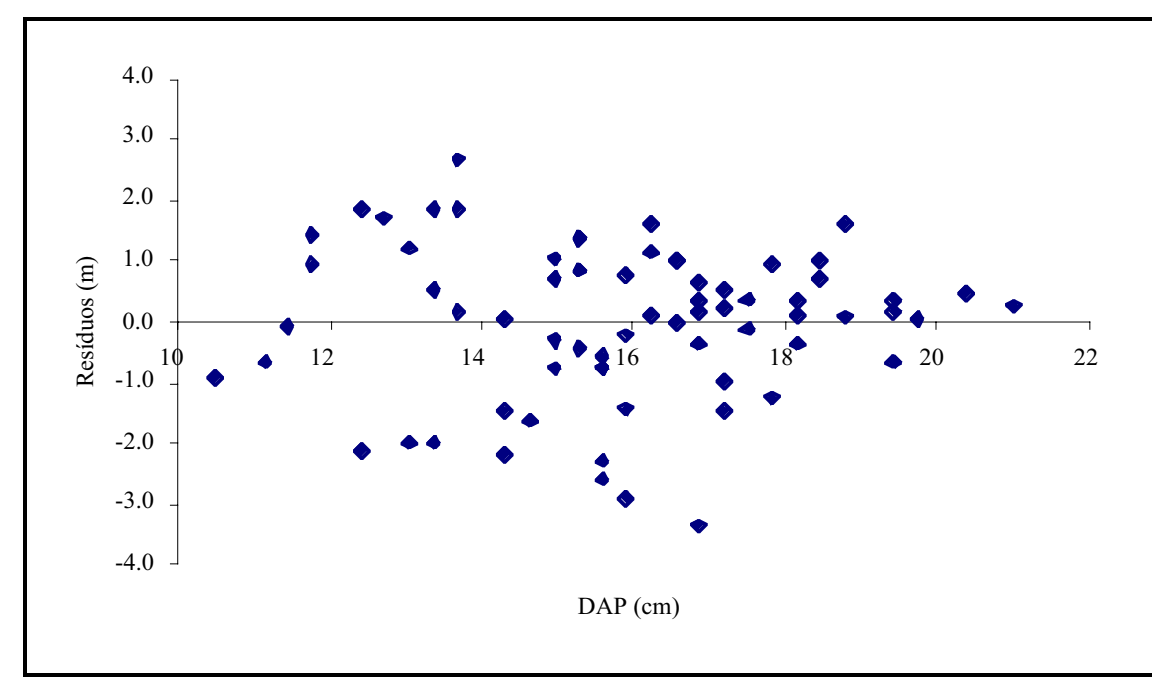


FIGURA 2 - Resíduos para altura observada e estimada com a equação 7

Figure 2 - Residues for the observed and estimated height using equation 7

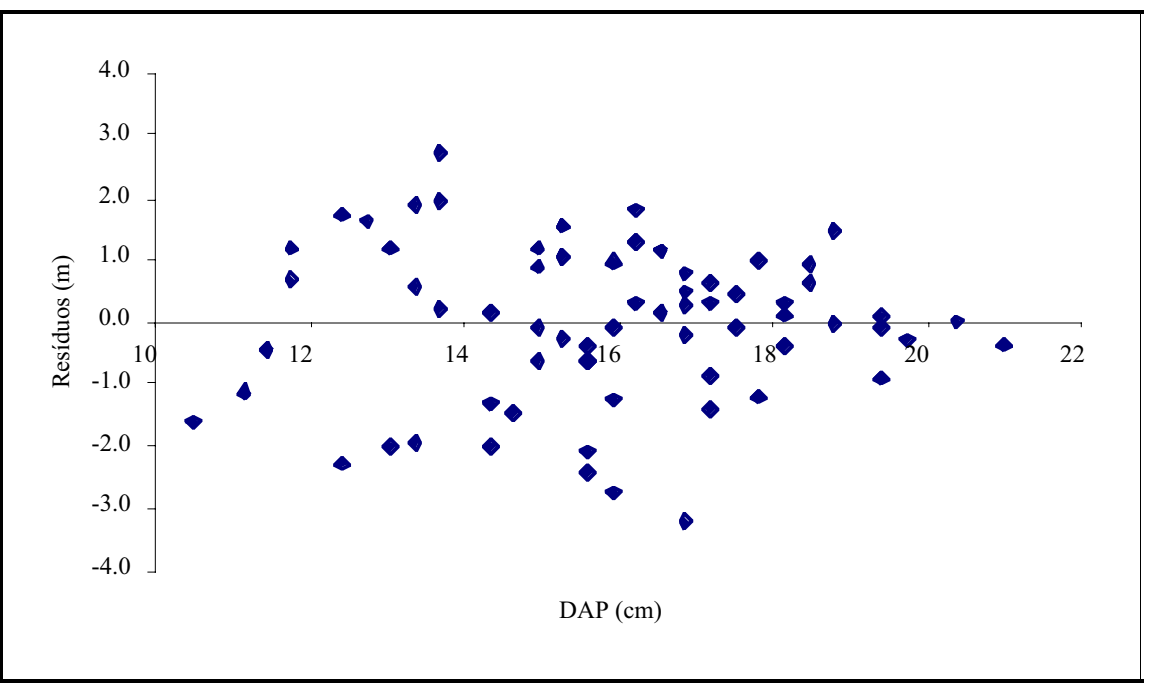

FIGURA 3 - Resíduos para altura observada e estimada com a equação 15

Figure 3 -Residues for the observed and estimated beight using equation 15

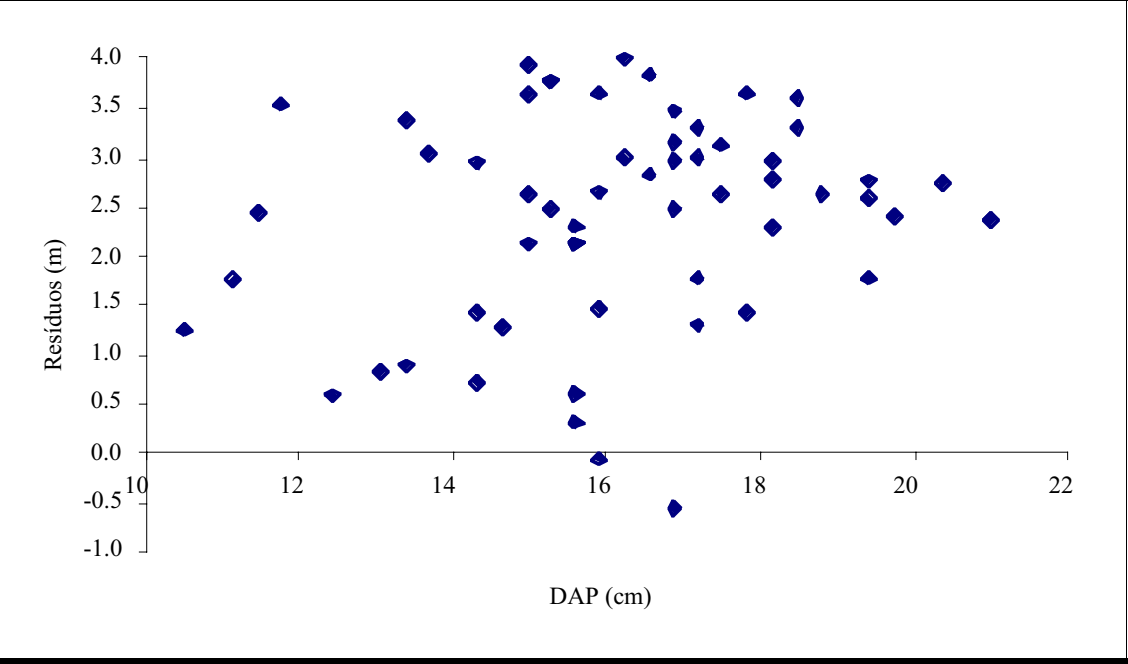

FIGURA 4 - Resíduos para altura observada e estimada com a equação16

Figure 4 - Residues for the observed and estimated beight using equation 16

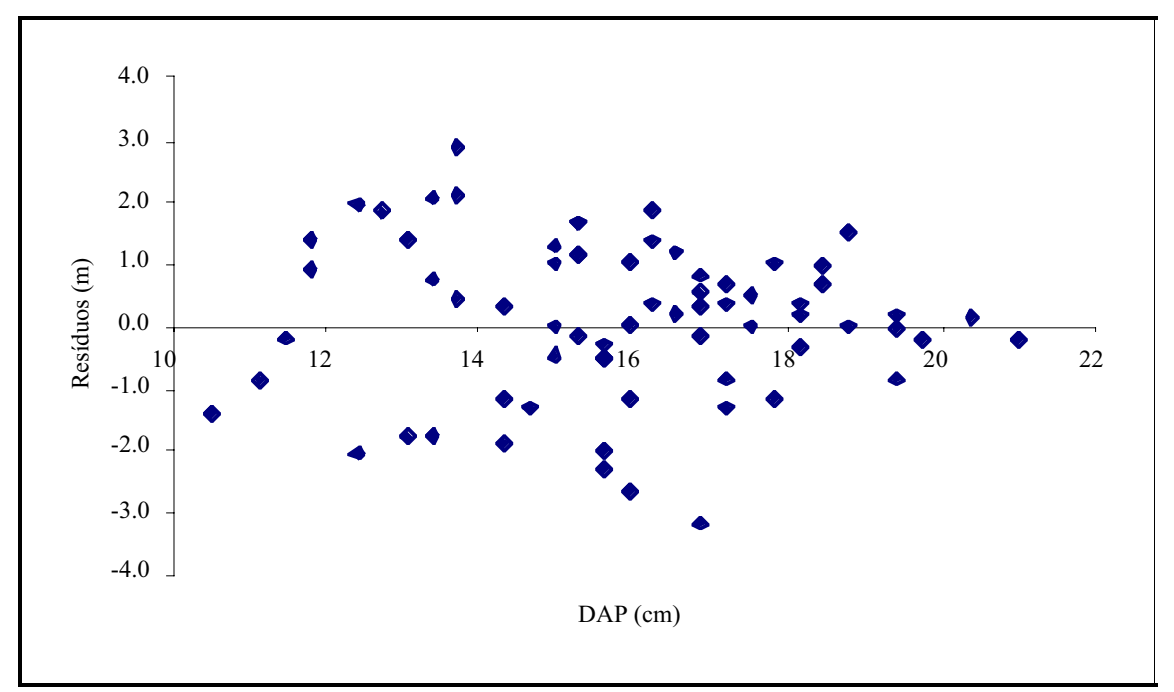


FIGURA 5 - Resíduos para altura observada e estimada com a equação 17

Figure 5 - Residues for the observed and estimated height using equation 17

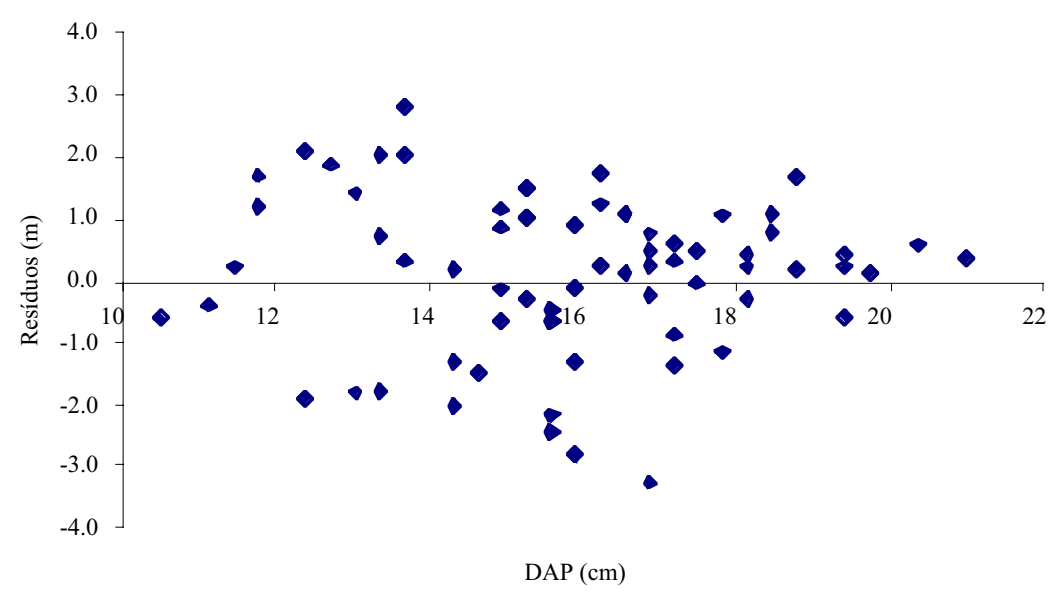

Com essas observações, percebe-se que a análise gráfica dos resíduos é uma ferramenta de grande importância para a seleção de modelos matemáticos de regressão, pois permitem uma melhor visualização da distribuição dos resíduos em todo o conjunto de dados. Pode-se separar, visualmente, as equações em dois grupos distintos, o que pode ser comprovado pela respectiva soma dos quadrados dos resíduos de cada equação. Na Figura 1, observa-se que as equações 5, 7, 16 e 17 apresentam amplitudes de resíduos semelhantes, o que é comprovado pela soma total de quadrados de resíduos de 110,2, 109,5, 111,1 e 112,0, respectivamente. A equação 15 apresenta uma situação bastante diferente, com soma total de quadrados de resíduos de 643,9.
A equação 17 apresentou uma boa distribuição de resíduos, muito similar a dos demais modelos, e sem tendências nítidas de superestimar ou subestimar as alturas dentro da amplitude de diâmetros observados. Dessa forma, com base no conjunto dos parâmetros estatísticos utilizados, conclui-se que a equação 17 apresenta um melhor ajuste dos dados de altura em função do diâmetro das árvores, sendo, por isso, selecionada e recomendada para fornecer estimativas de altura para povoamentos de araucária na região oeste do Paraná.

Na Figura 6, encontra-se a distribuição dos dados observados e a curva de alturas, ajustada com a equação 17, em função do diâmetro das árvores.

FIGURA 6 - Alturas observadas e estimadas para Araucaria angustifolia da região oeste do Estado do Paraná

Figure 6 - Observed and estimated heights for Araucaria angustifolia from the West Region of Parana State

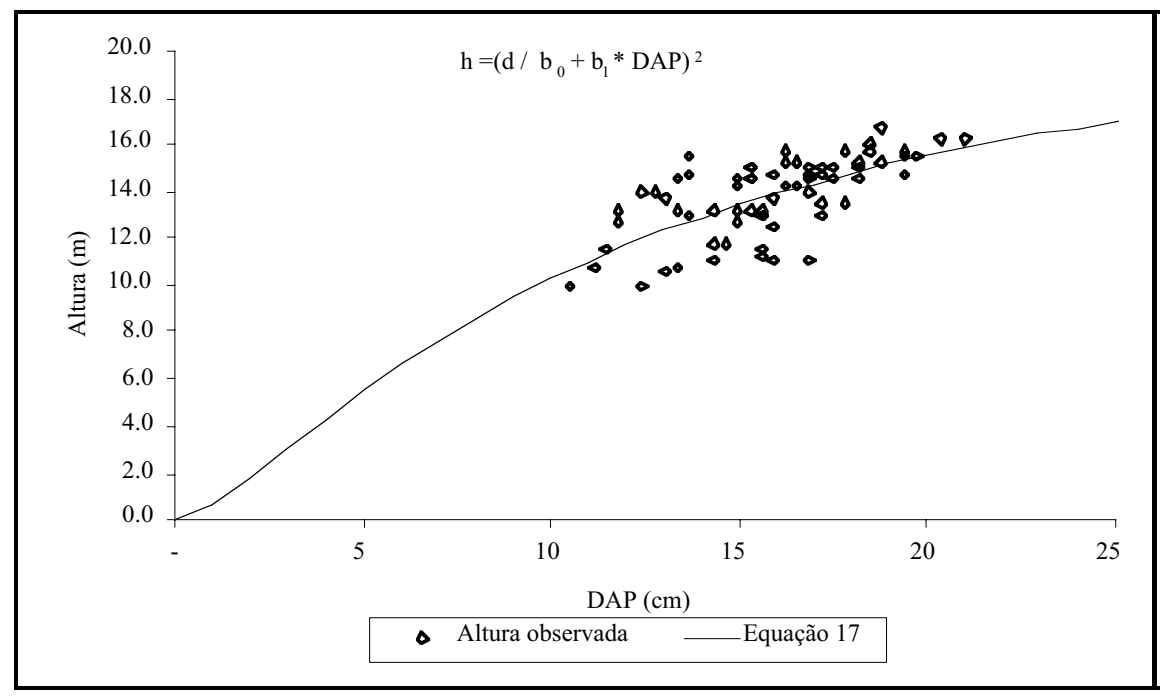




\section{Conclusões}

Com base nos resultados das análises estatísticas realizadas, pode-se concluir para o povoamento de Araucaria angustifólia, estudado no oeste do Paraná, o seguinte:

a) Cinco modelos matemáticos foram selecionados, de acordo com os critérios estatísticos, sendo que apenas o modelo 15 , representado pela equação $h-1,30=d^{2} /\left(b_{0}+b_{1}{ }^{*} d+b_{2}{ }^{*} d^{2}\right)$, apresentou tendência de subestimar as alturas em toda amplitude de diâmetros observados;

b) O modelo 17, representado pela equação $\mathrm{h}=\left(\mathrm{d} / \mathrm{b}_{0}+\mathrm{b}_{1}{ }^{*} \mathrm{~d}\right)^{2}$, apresentou melhor precisão estatística para o ajuste dos dados de altura em função do diâmetro, sendo recomendada sua utilização para a área de estudo.

\section{Referências}

AZEVEDO, C. P. et al. Relação hipsométrica para quatro espécies florestais em plantio homogêneo e em diferentes idades na Amazônia ocidental. Boletim de Pesquisa Floresta, Colombo, n.39, p.529, jul./dez., 1999.

CARVALHO, P. E. R. Espécies florestais brasileiras, recomendações silviculturais, potencialidades e uso da madeira. Colombo: Empresa Brasileira de Pesquisa Agropecuária - CNPF, 1994. $640 \mathrm{p}$.

GERHARD, E. J. Influência dos fatores físicos do solo e dos nutrientes da serapilheira sobre o crescimento em altura da Araucaria angustifolia (Bert,) O. Kuntze, Santa Maria: UFSM, 1999. 98f. Dissertação (Mestrado em Ciências Florestais) -Universidade Federal do Paraná, 1999.

HUECK, K. As florestas da América do Sul. São Paulo: Polígono, 1972. p.206-239, 466p.

IPAGRO. Seção de Ecologia Agrícola. Atlas agroclimático do Estado do Rio Grande do Sul. Porto Alegre, 1989. 3v.

JARDIM, F. C. S. Estrutura da floresta equatorial úmida da Estação Experimental de Silvicultura Tropical, Manaus. INPA - FUA, 1985. $198 f$. Dissertação (mestrado) - Instituto Nacional de Pesquisa da Amazônia, 1985.
LOETSCH, F.; ZOHRER, F.; HALLER, K. E. Forest Inventory, Bonn: [s.n], 1973. v,2. 469 p.

MACHADO, S. A.; BASSO, S. F.; BEVILACQUA JÚNIOR, V. G. Teste de modelos matemáticos para o ajuste da relação hipsométrica em diferentes sítios e idades para plantações de Pinus elliottii no Estado do Paraná, In: CONGRESSO FLORESTAL BRASILEIRO, 7, 1993, Curitiba. Anais... São Paulo: SBS, 1993, v.2, p.553-556.

MOREnO, J. A. Clima do Rio Grande do Sul. Porto Alegre: Secretaria da Agricultura, 1961. 42p.

REITZ, R.; KLEIN, R. M.; REIS, A. Projeto madeira do Rio Grande do Sul. Sellowia, Itajaí, n.34/35, 525 p. 1983.

SCHIMIDT, P. B. Determinação indireta da relação hipsométrica para povoamentos de Pinus taedh, Curitiba: UFPR, 1977. 102f. Dissertação (Mestrado em Ciências Florestais) - Universidade Federal do Paraná, 1997.

SCHNEIDER, P. R. Introdução ao manejo florestal. Santa Maria: CEPEF/FATEC, 1993. 320p.

SCOLFORO, J. R. S. Biometria Florestal 2, Técnicas de regressão aplicada para estimar: volume, biomassa, relação hipsométrica e múltiplos produtos de madeira, UFLA/FAEPE/DCF, 1997. 292p.

SILVA, J. N. M. Relação hipsométrica de espécies da Floresta Nacional do Tapajós, Belém: EMBRAPA - CPATU, 1980. 2p.

ZANON, M. L. B.; FINGER, C. A. G.; SCHNEIDER, P. R. Funções para descrever a relação altura e diâmetro de Eucalyptus dunnii Maiden. Ciência Rural, Santa Maria, v.26, n.1, p.87-90, 1996.

Recebido 25/2/2002 Aprovado 14/3/2003 